Check for updates

Cite this: J. Mater. Chem. A, 2018, 6, 7538

Received 29th November 2017 Accepted 25th March 2018

DOI: $10.1039 / c 7 t a 10509 f$

rsc.li/materials-a

\section{Proton jump diffusion dynamics in hydrated barium zirconates studied by high-resolution neutron backscattering spectroscopy $\dagger$}

\author{
Daria Noferini, (D) ${ }^{\text {ab }}$ Bernhard Frick, (D) ${ }^{\mathrm{b}}$ Michael Marek Koza (D) ${ }^{\mathrm{b}}$ \\ and Maths Karlsson (D) *a
}

\begin{abstract}
We report results from high-resolution neutron backscattering experiments on hydrated samples of the proton conducting perovskite $\mathrm{BaZr}_{1-x} \mathrm{M}_{x} \mathrm{O}_{3-x / 2}$ with $\mathrm{M}=\mathrm{Sc}$ and $\mathrm{Y}$ for $x=0.1$, and $\mathrm{M}=\ln$ for $x=0.1,0.2$ and 0.25. The sampled wave vector range of up to $1.9 \AA^{-1}$ and energy resolution of less than $1 \mu \mathrm{eV}$ allowed the identification of a jump diffusion process of protons in the samples. By monitoring the intensity of elastically scattered neutrons $S(Q, \hbar \omega=0)$ upon heating the samples from base temperature $2 \mathrm{~K} \mathrm{up}$ to about $550 \mathrm{~K}$ the onset temperature $T_{\mathrm{c}}$ of the diffusive process was established. Thereby characteristic dependences of $T_{\mathrm{c}}$ on the type of dopant atom $M$ and on the dopant level $x$ were found with $T_{c}$ increasing along the sequence of $x$ from less than $200 \mathrm{~K}$ in $M=S c, Y$ and $\ln$ with $x=0.1$ up to about $320 \mathrm{~K}$ in $M=\ln$ with $x=0.25$. The formfactor of the diffusive process was examined on one hand by monitoring the intensity of neutrons scattered inelastically with a fixed energy of $2 \mu \mathrm{eV}$ as $S(Q, \hbar \omega=$ $2 \mu \mathrm{eV})$, and on the other, by monitoring the dynamic structure factor $S(Q, \hbar \omega)$ within the dynamic range of $\pm 25 \mu \mathrm{eV}$. We have successfully approximated the data by established models of jump diffusion with a preference for the Chudley-Elliot model [C. T. Chudley, R. J. Elliot, Proc. Phys. Soc., 77, 353, (1961)]. Any of the applied models featured a characteristic jump distance of about $3 \AA$. Diffusion constants $D$ and activation energies $E_{\mathrm{a}}$ were computed from temperature scans of the moderately $\mathrm{In}$-doped compounds $\left(x=0.1\right.$ and 0.2 ). $D$ takes on values within the range $1-5 \times 10^{-7} \mathrm{~cm}^{2} \mathrm{~s}^{-1}$ at the highest applied $T$ of about $550 \mathrm{~K}$ and $E_{\mathrm{a}}$ increases from about 40 to $120 \mathrm{meV}$ along the sequence of $x$.
\end{abstract}

\section{Introduction}

Acceptor-doped proton conducting perovskites, such as Indoped $\mathrm{BaZrO}_{3}$ (BZO), are currently receiving considerable attention because of their promising application as electrolytes in next-generation fuel cells and hydrogen separation membranes. ${ }^{1}$ A critical, long-standing, challenge pertains to understanding and controlling the level of proton conductivity by modification of the (local) structure of the perovskite lattice, as influenced by, e.g., the type and concentration of acceptor dopants. ${ }^{2,3}$

The current understanding of the proton transport mechanism in proton conducting oxides is based primarily on results obtained from computer simulations, and from quasielastic neutron scattering (QENS) on bulk powder samples of acceptordoped $\mathrm{BaZrO}_{3}$ and $\mathrm{BaCeO}_{3}$, as summarized recently. ${ }^{4}$ We showed in a series of preceding QENS experiments based on the

${ }^{a}$ Department of Chemistry and Chemical Engineering, SE-412 96 Göteborg, Sweden. E-mail: maths.karlsson@chalmers.se; Tel: +46 733873207

${ }^{b}$ Institut Laue-Langevin, CS 20156, 38042 Grenoble Cedex 9, France

$\dagger$ Electronic supplementary information (ESI) available. See DOI: $10.1039 / \mathrm{c} 7 \mathrm{ta} 10509 \mathrm{f}$ time-of-flight (ToF) technique, that the response of the local dynamics of protons in Sc-, Y- and In-doped BZO is reminiscent of a jump motion between two and four equivalent sites..$^{5-7}$ These dynamics correspond to translations of protons between two adjacent oxygens and $\mathrm{O}-\mathrm{H}$ reorientations, respectively. To access the required momentum transfers $(Q)$ for monitoring the local dynamics the resolution of the spectrometers had to be relaxed to $0.2-0.6 \mathrm{meV}$. Only very fast relaxation dynamics on the order of picoseconds and thus in the vicinity of low-energy vibrations could have been examined that way. An extension of the experimentally accessible timescales into the regime of nanoseconds was attempted with the use of the QENS neutron spin-echo (NSE) technique. ${ }^{8-10}$ For hydrated samples of Sc-, Y-, and In-doped BZO the response showed a clear relaxation, however, not specific to a well defined process characterized by a unique activation energy but resulting from a distribution of activation energies underlying the relaxation mechanism. A drawback of the NSE technique applied to the specific scattering properties of the samples was the unfavourable ratio of coherent to incoherent scattering powers cancelling out the interpretable signal in an extended $Q$-range. A $Q$-range of $0.8-1.2 \AA^{-1}$ could be exploited only, which did not allow for an examination of the spatial geometry of the observed relaxation processes. 
With the present study we intend to overcome this limitation and to examine the relaxation channels of Sc-, Y-, and In-doped BZO on timescales of up to about one nanosecond and with a sufficiently large $Q$-range to identify the spatial geometry of the dynamics. The experimental tool applied here is the neutron backscattering (NBS) technique, which offers a resolution of less than $1 \mu \mathrm{eV}$, i.e. it covers a time domain very comparable to the one sampled in NSE measurements, with an exploitable $Q$-range of up to $1.9 \AA^{-1}$, i.e. approaching the $Q$-range in our preceding ToF experiments. ${ }^{5-7}$ The detriment of NBS in comparison to NSE is the somehow reduced neutron flux on the sample making the characterization of some of the relaxation signals challenging, as we show hereafter.

\section{Experimental}

Hydrated samples of the compositions $\mathrm{BaZr}_{1-x} \mathrm{M}_{x} \mathrm{O}_{3-x / 2}$ with $\mathrm{M}=\mathrm{Sc}$ and $\mathrm{Y}$ for $x=0.1$ (10Sc:BZO and 10Y:BZO), and $\mathrm{M}=\mathrm{In}$ for $x=0.1,0.2$ and 0.25 (10In:BZO, 20In:BZO, and 25In:BZO) were studied. These samples are the very same as characterized and studied in our preceding ToF and NSE measurements. Details about the preparation and characterization of the samples by different techniques can be extracted from ref. 6, 7 and 10. Their hydrogen concentration, as determined from neutron diffraction with polarization analysis, are $0.34(3)$ (10Y:BZO), 0.18(2) (10Sc:BZO), 0.10(1) (10In:BZO), $0.20(1)$ (20In:BZO), and 0.19(1) (25In:BZO) $\mathrm{mol} \mathrm{mol}_{\mathrm{Ba}}{ }^{-1} \cdot 6,7,10$

The NBS experiments were conducted at the IN16B spectrometer of the Institut Laue Langevin (ILL) in Grenoble, France. ${ }^{11,12}$ With an incident wavelength of $6.3 \AA$ and the Si(111) unpolished analyzer configuration a resolution of $0.9 \mu \mathrm{eV}$ and a maximum $Q$ of $1.9 \AA^{-1}$ were accessed. IN16B was operated in three different modes. First, we examined the intensity in the elastic channel at $\hbar \omega=0$ (elastic fixed window scan; EFWS) and the inelastic channel $\hbar \omega=2 \mu \mathrm{eV}$ (inelastic fixed window scan; IFWS) of all listed samples upon heating from the base temperature $T \approx 2 \mathrm{~K}$ up to about $550 \mathrm{~K}$. The undoped material (BZO), was as well examined in the EFWS mode as the reference sample not exhibiting relaxation processes within the studied $T$ range. By this approach, data on the evolution of the isotropic thermal displacement parameters $U_{\text {iso }}(T)$, on the onset temperatures $T_{\mathrm{c}}$ of diffusive dynamics and on the intensity of the associated signal in the inelastic regime were gathered. Based on this information, scans of the dynamic structure factor $S(Q, \hbar \omega)$ were programmed within the dynamic range of $\pm 25 \mu \mathrm{eV}$. The neutron flux in this dynamic scan mode is reduced by about two orders of magnitude in comparison to the EFWS and IFWS modes and made it necessary to select temperatures for the most promising QENS signal. Thus, the applied $T$ differ between the samples and are reported with the data hereafter.

We applied standard corrections to the data comprising empty can scattering, detector efficiency adjustments and absorption evaluations. A comparison of the base $T$ sample data with a monitored vanadium standard confirmed the equivalence of the signals. As a consequence and for reasons of best statistical accuracy the presented data were thus normalized to the respective EFWS signals of each specimen, averaged in the low- $T$ range of $2-20 \mathrm{~K}$. This approach is very well justified as the recorded signal is dominated by the incoherent scattering of the protons in the samples and all dynamics are frozen out at the base temperature. ${ }^{\mathbf{1 0}}$ The resolution function required for the analysis of $S(Q, \hbar \omega)$ was computed from the response of the 20In:BZO compound, which showed the strongest signal, at base $T \approx 2 \mathrm{~K}$. However, it is not applicable to signals containing intensities from Bragg reflections of the coherently scattering BZO matrix. Following the approach of our preceding studies, $Q$ regions with Bragg peak contributions were excluded from the analysis of the data. All basic corrections of the data and conversions of the raw signal to $S(Q, \hbar \omega)$ were performed with the software package LAMP supplied by the ILL. ${ }^{13}$ The fitting procedures were carried out with routines and $\chi^{2}$ minimization algorithms of the software packages LAMP, MATLAB and Gnuplot. ${ }^{13-15}$

\section{Results}

\subsection{Elastic fixed window scans}

We report in Fig. 1 the logarithm of the relative elastic intensities $\ln [I(Q, T) / I(Q, 2 \mathrm{~K})]$ as a function of $Q^{2}$ and $T$. For solids displaying solely harmonic dynamics this intensity can be evaluated and the observable approximated as

$$
\ln [I(Q, T) / I(Q, 2 \mathrm{~K})] \approx-\Delta U_{\mathrm{iso}}(Q, T) Q^{2}
$$

with $\Delta U_{\text {iso }}(Q, T)=U_{\text {iso }}(Q, T)-U_{\text {iso }}(Q, 2 \mathrm{~K})$ being the isotropic thermal displacement parameter corrected for its base temperature value. ${ }^{\mathbf{1 6 , 1 7}}$ The linear dependence of $\ln [I(Q, T) / I(Q, 2$ K)] upon $Q^{2}$ can be exploited to compute $\Delta U_{\text {iso }}(Q, T)$ which for a solid displaying a purely harmonic dependency of the

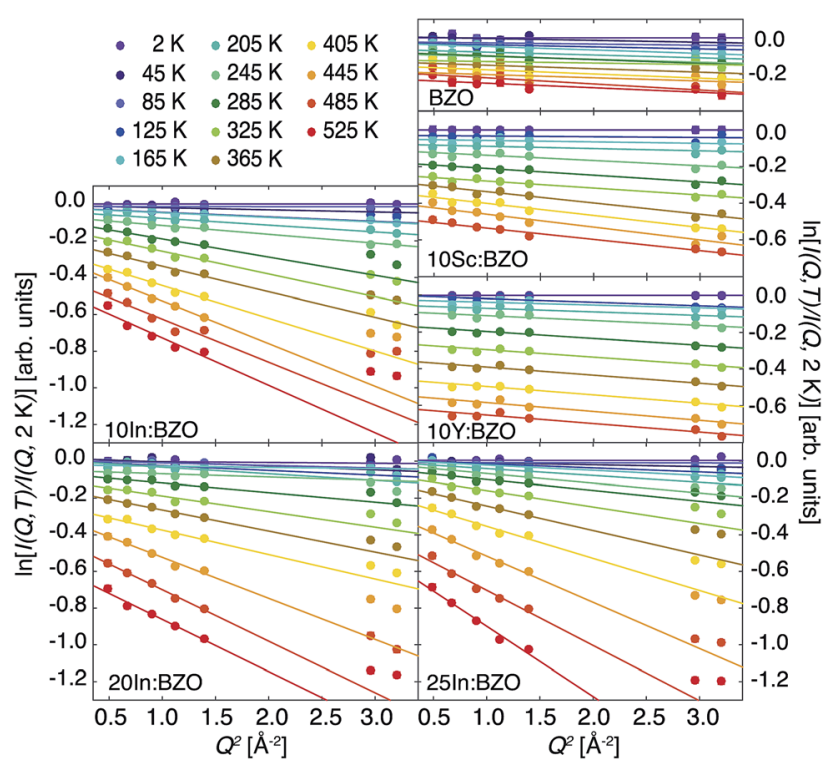

Fig. 1 Relative elastic intensities $\ln [/(Q, T) / /(Q, 2 K)]$ measured in the EFWS mode. Results of fits with a linear dependence upon $Q^{2}$ up to $1.4 \AA^{-2}$ (In-doped series) or $1.9 \AA^{-2}$ (BZO, 10Sc:BZO, and 10Y:BZO) are plotted as lines. 
dynamics is supposed to follow $T$ linearly at sufficiently high $T .^{\mathbf{1 8}}$ Any relaxation channel activated by temperature leads to a departure from the linear dependence of $\ln [I(Q, T) / I(Q, 2 \mathrm{~K})]$ upon $Q^{2}$. However, a linear regime can be always found and approximated towards smaller $Q$, which is known as the Gaussian approximation. ${ }^{19} \Delta U_{\text {iso }}(Q, T)$ approximated within the Gaussian approximation will then show a departure from the linear $T$ dependence deviating towards higher values than expected from the vibrational properties of a solid showing a harmonic behaviour. The temperature points $T_{\mathrm{c}}$ at which the linearities in $\ln [I(Q, T) / I(Q, 2 \mathrm{~K})] \propto Q^{2}$ and $\Delta U_{\text {iso }}(Q, T) \propto T$ break down are thus characteristic of the onset of relaxation in the acceptor-doped BZO materials. Since, in any of the samples studied here more than $80 \%$ of the total signal is related to protons we can safely state that this break down is related to proton relaxation.

We fitted the linear dependence in the entire $Q$ range and within the Gaussian approximation up to $Q^{2}=1.4 \AA^{-2}$. For BZO, 10Sc:BZO, and 10Y:BZO the linear dependence is well respected up to the highest $Q^{2} \approx 3 \AA^{-2}$ indicating an apparent harmoniclike character of the response up to the highest $T$. For the Indoped materials a mismatch of the high $Q$ points with the linear dependence is obvious for $T$ higher than about $200 \mathrm{~K}$. We highlight this mismatch by plotting in Fig. 1 results of the Gaussian approximation.

The derived $\Delta U_{\text {iso }}(Q, T)$ are reported in Fig. 2. As expected, all samples show increasing thermal parameters upon heating. In accordance with the behaviour of $\ln [I(Q, T) / I(Q, 2 \mathrm{~K})]$ there is an

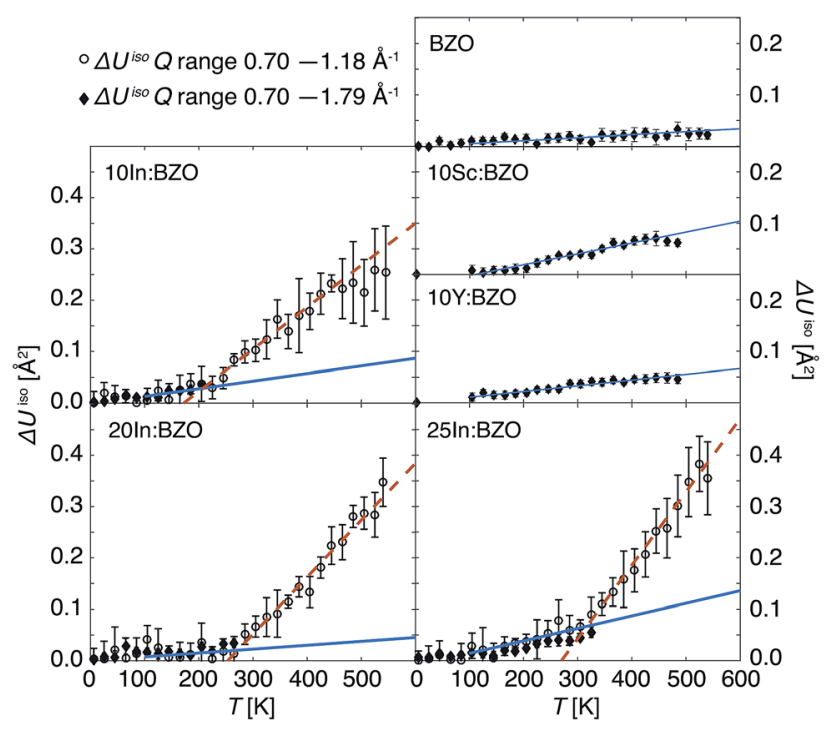

Fig. 2 Thermal displacement parameters $\Delta U_{\text {iso }}(Q, T)$ derived from the relative intensities reported in Fig. 1. Black open circles correspond to results obtained in the entire $T$ range within the Gaussian approximation up to $Q^{2}=1.4 \AA^{-2}$. Black filled diamonds represent results derived within the harmonic $T$ regime of the compounds by accounting for the entire $Q$ range. For the In-doped materials, such results are reported up to $T_{\mathrm{c}}$; at higher temperatures, the linearity of In $[/(Q, T) / /(Q, 2 K)] \propto Q^{2}$ does not hold for $Q^{2}>1.4 \AA^{-2}$. Solid blue and dashed red lines plotted with the experimental data indicate the results of linear fits according to eqn (3) highlighting the two respective slopes $m_{1}$ and $m_{2}$. enhanced deviation of the $\Delta U_{\text {iso }}(Q, T)$ towards higher values in the In-doped materials above about $200 \mathrm{~K}$. Thus, both the $Q$ dependence of the relative intensities as well as the $T$ dependence of the thermal displacement parameters give evidence of an additional relaxation channel setting in above $200 \mathrm{~K}$ in the In-doped materials.

To set this observation onto quantitative grounds, $\Delta U_{\text {iso }}(Q, T)$ was parametrized with the following function

$$
\begin{gathered}
f(T)=c+m_{1} T \text { for } 100 \mathrm{~K} \leq T<T_{\mathrm{c}} \\
f(T)=c+m_{1} T_{\mathrm{c}}+m_{2}\left(T-T_{\mathrm{c}}\right) \text { for } T \geq T_{\mathrm{c}}
\end{gathered}
$$

and the parameters $c, m_{1}, m_{2}$ and $T_{\mathrm{c}}$ determined by a $\chi^{2}$ fit to the data. Here, $c$ and $m_{1}$ approximate the high $T \geq 100 \mathrm{~K}$ behaviour in the harmonic regime of the solids. The choice of a linear dependence with slope $m_{2}$ above $T_{\mathrm{c}}$ was applied for reasons of convenience without physical justification, which would require the knowledge of the microscopic relaxation mechanism which has not been established, yet. No clear change in the dynamics of BZO, 10Sc:BZO and 10Y:BZO could be established, although, for the two doped materials, a trend towards enhanced $\Delta U_{\text {iso }}(Q$, $T$ ) uptake could be conjectured with a $T_{\mathrm{c}}$ of even less than $200 \mathrm{~K}$. As we outline later, this apparent harmonic behaviour in the entire $Q$ range is only a consequence of a too low contrast between the QENS and elastic signals.

We report the fit parameters in Fig. 3 . The slope $m_{1}$ within the harmonic regime is very comparable in all compounds which might find its reason in akin vibrational properties. Approximations with a unique $m_{1}$ for all samples were successful with an insignificant effect on $m_{2}$ and $T_{\mathrm{c}}$. These two parameters show a clear dependence on the doping ratio trending towards higher values for higher In dopant levels. With the present resolution of $0.9 \mu \mathrm{eV}$ the onset $T_{\mathrm{c}}$ of diffusion is identified as low as about $200 \mathrm{~K}$ in 10In:BZO and we conclude that the harmonic thus purely vibrational dynamics persist in each material up to this temperature. To highlight this point we compare in Fig. 2 $\Delta U_{\text {iso }}(Q, T)$ data up to $T_{\mathrm{c}}$ approximated in the entire $Q$ range of $\ln$ $[I(Q, T) / I(Q, 2 \mathrm{~K})]$ and within the Gaussian approximation. They are identical within the statistical accuracy.

For a material displaying a harmonic behaviour the high $T$ slope $m_{1}$ can be related to some characteristic energy such as

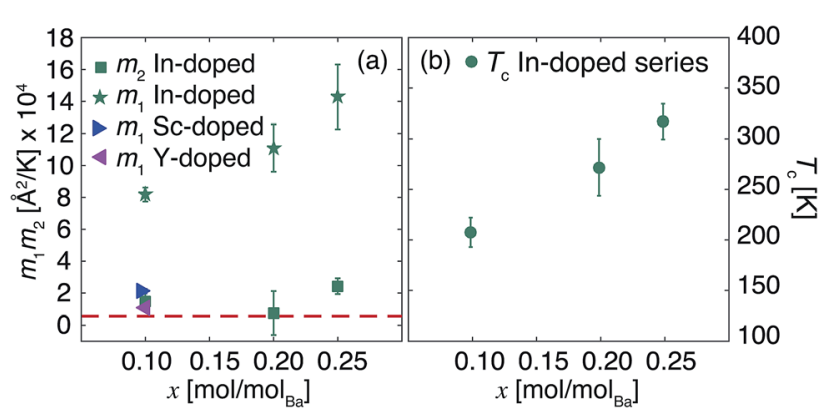

Fig. 3 Parameters $m_{1}, m_{2}$ (a) and $T_{c}$ (b) derived from fits of eqn (3) to $\Delta U_{\text {iso }}(Q, T)$ reported in Fig. 2. The dashed red line in panel (a) highlights the $m_{1}$ value for the undoped material (BZO). When not displayed, errors are within the symbols or the thickness of the line. 
the Einstein energy $\hbar \omega_{\mathrm{E}}$ within the Einstein model of solids $\bar{M}\left(\hbar \omega_{\mathrm{E}}\right)^{2}=k_{\mathrm{B}} \hbar^{2} m_{1}{ }^{-1} .^{18}$ The interpretation of $m_{1}$ is hampered here by the response of an unknown effective mass $\bar{M}$. Let us consider however two specific cases such as $\bar{M}$ being the mass of a proton. In this case $\hbar \omega_{\mathrm{E}}$ is in the range of $40-50 \mathrm{meV}$, a reasonable value in view of a wide spread of vibrational frequencies hydrogen contributes to in hydrated BZO materials. On the other hand, we may consider the first localized vibrational states leading to pronounced peaks in the vibrational density of states at energies of $10-20 \mathrm{meV}$ (ref. 7) as some characteristic $\hbar \omega_{\mathrm{E}}$. In this case the effective mass $\bar{M}$ approaches the mass of the $\mathrm{OH}$ group. Thus, $m_{1}$ takes on physically reasonable measures.

\subsection{Inelastic fixed window scans}

Selected $T$ - and $Q$-dependent signals registered in IFWS mode at $\hbar \omega=2 \mu \mathrm{eV}$ are reported in Fig. 4. The inelastic signal is normalized to the corresponding elastic line intensity at the base temperature as $I(Q, 2 \mu \mathrm{eV}, T) / I(Q, 0,2 \mathrm{~K})$. At $T \leq 100 \mathrm{~K}$ no signal in the inelastic channel is expected, thus, an averaged background intensity was subtracted from the data sets for convenience. In accordance with the conclusions drawn from the EFWS data an onset of relaxation processes is evidenced by the uptake of the IFWS signal at elevated $T$. Because of the higher signal contrast in the off-elastic line channel this uptake is indicated already below $200 \mathrm{~K}$, and it is visible also for 10Y:BZO and 10Sc:ZBO.

Three prominent features can be seen in the IFWS data: (i) the amplitude of the inelastic signal increases with the sequence of Sc-, Y-, and In-doped compounds as well as with the increase of the nominal doping level of the In-doped series. As the signal reflects the intensity of the proton relaxation relative to the total number of protons present in the samples it gives evidence of a higher propensity of protons to diffuse for the Indoped samples and along the sequence of an increasing dopant level. (ii) The evolution of the IFWS intensity upon $T$ changes and the signal profile is not universal. For 10Sc:BZO and

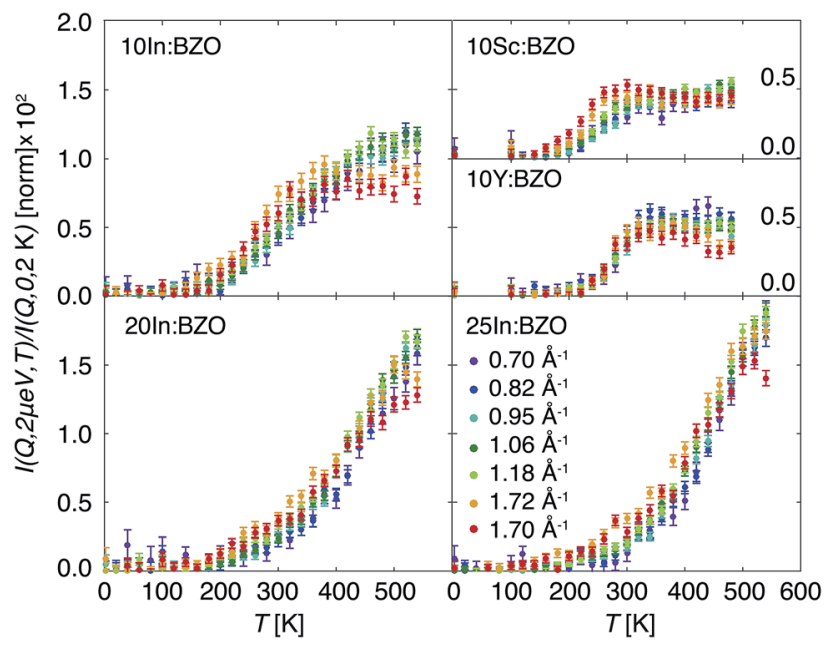

Fig. 4 Intensities $/(Q, 2 \mu \mathrm{eV}, T) /(Q Q, 0,2 \mathrm{~K})$ of the hydrated barium zirconate compounds measured with the IFWS mode.
10Y:BZO the response passes through a distinct maximum in the $T$ range of 250-400 K, a trend also observed for 10In:BZO. It displays, however, at higher $Q$ a plateau of intensity above $350 \mathrm{~K}$ rather than a distinguished maximum. For 25In:BZO the signal increases monotonically within the entire $T$ regime of relaxation showing a weak saturation effect at the highest $T$ only. The intermediate In-doped material shows an evolution anticipated from the intermediate stages between 10In:BZO and 25In:BZO. (iii) A less obvious feature in the response of the $10 \% \mathrm{Sc}-, \mathrm{Y}-$, and In-doped materials is a dependence of the maximum on the wave vector $Q$. Particularly well visible in 10Sc:BZO, this maximum appears to be shifted to lower $T$ for higher $Q$ indicating that traces of non-localized, diffusive dynamics are picked up at this early stages of proton relaxation by the highresolution experiment. For localized dynamics this maximum should appear at identical $T$ for different $Q .^{12,20,21}$ From the present data, it is not readily at hand to draw this conclusion for the higher doped In-containing materials as the signal does not reach a maximum in the examined $T$ range. A diffusive response may not be discarded for the same reason. Nonetheless, up to about $20 \%$ In doping the onset of a saturation effect can be observed for the high- $Q$ IFWS data at high $T$.

To highlight the effect of a basic jump diffusion process on the IFWS response we report in Fig. 5 an IFWS signal computed with a normalized standard Lorentzian function $L(Q, \hbar \omega)$ characterizing the QENS signal whose width $\Gamma$ is determined by the Chudley-Elliot model (CEM) ${ }^{22}$ of jump diffusion defined as

$$
\frac{\Gamma}{2}=\frac{\hbar}{\tau}\left(1-\frac{\sin \left(Q l_{\mathrm{CEM}}\right)}{Q l_{\mathrm{CEM}}}\right),
$$

with a characteristic jump distance $l_{\text {CEM }}$, and a mean residence time $\tau$. The displayed data were computed with $l_{\text {CEM }}=3.0 \AA$, motivated by results discussed hereafter, and $\tau$ approximated by an Arrhenius $T$-activated process with the activation energy $E_{\mathrm{a}}=$ $100 \mathrm{meV}$ and the trial period $\tau_{\mathrm{o}}=10 \mathrm{ps}$. These parameters were derived in our preceding experiments on 10Sc:BZO and 10Y:BZO. ${ }^{6}$

With the chosen parameters the main features observed particularly well in the response of 10Sc:BZO are reproduced in the modelled IFWS signal; the presence of a peak in the temperature range of 300-400 $\mathrm{K}$, a shift of this peak towards lower $T$ for higher $Q$, and the take-off of the IFWS signal already

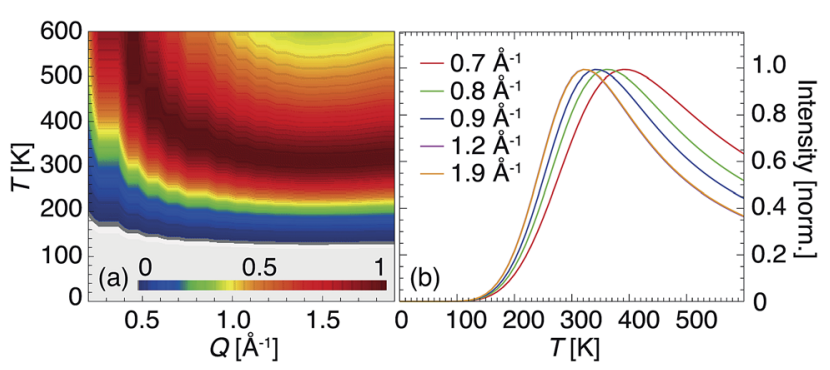

Fig. 5 IFWS signal computed for the $Q$ range accessible on IN16B. Contrast plot of the IFWS response highlighting the maximum dependence on $Q$ and $T$ up to $600 \mathrm{~K}$ (a). Selected profiles at $Q$ specific for the present experiment plotted in Fig. 4 (b). 
below $200 \mathrm{~K}$. However, this approach does not reproduce the profile exactly towards high temperatures captured in Fig. 4. An additional increase in intensity gives evidence of additional relaxation channels activated at elevated $T$. The experimental signal is not sufficiently specific to judge upon its origin, i.e. whether it is of localized or diffusive character.

It is necessary to highlight here that the experimental data visualized as $I(Q, 2 \mu \mathrm{eV}, T) / I(Q, 0,2 \mathrm{~K})$ in Fig. 4 are not corrected for the Debye-Waller factor. The moderate reduction of intensity of the maximum in the IFWS signal for higher $Q$ towards high $T$ is expected and should not be mistaken for a characteristic feature. This does not apply to the modeled response as shown in Fig. 5.

\subsection{Dynamic structure factor}

In Fig. 6 we present selected spectra of the dynamic structure factor $S(Q, \hbar \omega, T) / S^{\prime}(Q, 0,2 \mathrm{~K})$, with $S^{\prime}(Q, 0,2 \mathrm{~K})$ the EFWS signal, of 20In:BZO at the applied temperatures of 350, 450 and $550 \mathrm{~K}$. A parametrization of the data is carried out with the following equation

$S(Q, \hbar \omega)=\left[a_{\mathrm{D}}(Q) \delta(\hbar \omega)+a_{\mathrm{L}}(Q) L(Q, \hbar \omega)\right] \times R(Q, \hbar \omega)+b(Q)$

with $a_{\mathrm{D}}(Q) \delta(\hbar \omega)$ characterizing the elastic intensity, $a_{\mathrm{D}}(Q) L(Q$, $\hbar \omega)$ representing a single Lorentzian response of the QENS signal, both convoluted with the measured resolution of the spectrometer $R(Q, \hbar \omega)$ and a flat, $\hbar \omega$-independent, background $b(Q)$.

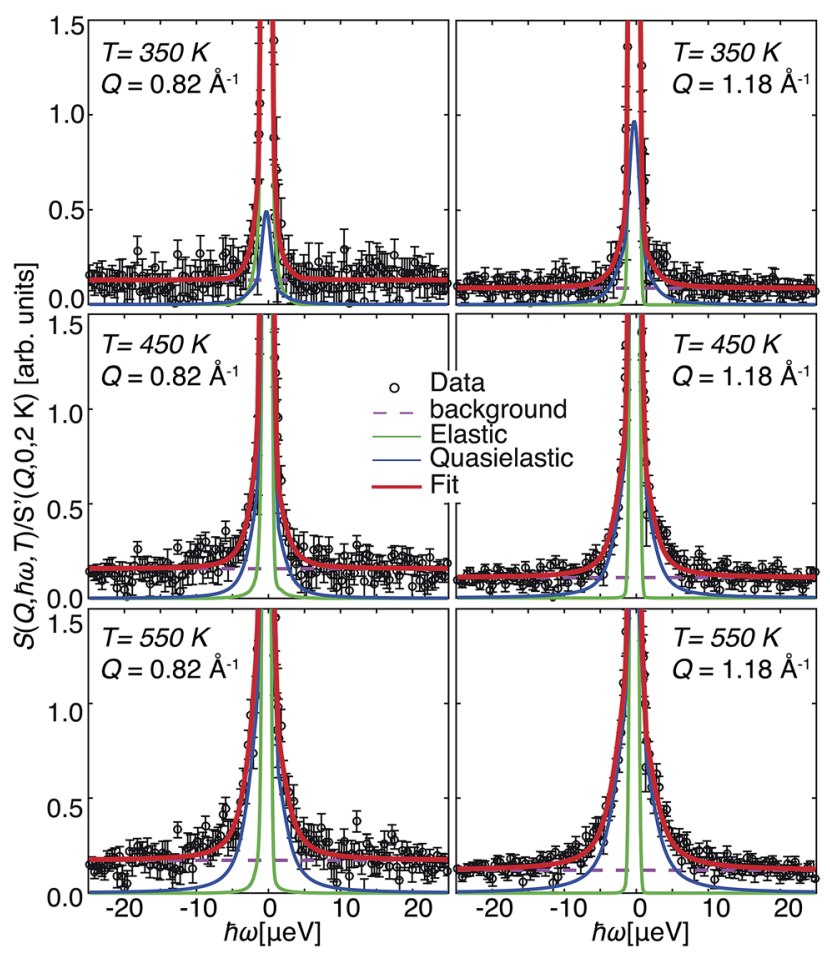

Fig. 6 Dynamic structure factor $S(Q, \hbar \omega, T) / S^{\prime}(Q, 0,2 K)$ of 20 In: $B Z O$ for two selected $Q$ and all applied $T$. Lines represent fit results with eqn (5).
The statistical significance of the data is sufficient only for 20In:BZO to carry out a free-parameter analysis of the spectra with eqn (5). Results from the free-parameter fit are shown in Fig. 7(a) and (b). The $Q$-independent intensity in Fig. 7(a) and the ascending Lorentzian full widths at half maximum $\Gamma$ in Fig. 7(b) are attributes of a diffusive process to the QENS response. This process becomes faster upon heating evidenced by the enhanced $\Gamma$ at higher $T$.

The same features can be conjectured from the response of 10In:BZO at the highest $T$. However, the analysis and interpretation of any other data sets require the application of model assumptions. Even in the case of 20In:BZO at $T \approx 350 \mathrm{~K}$ the refinement of the geometrical characteristics of the relaxation process profits from restricting the intensities to $Q$-independent values, i.e. $a_{\mathrm{D}}(Q)=\varepsilon a_{\mathrm{D}}$ and $a_{\mathrm{L}}(Q)=(1-\varepsilon) a_{\mathrm{D}}$. The parameter $\varepsilon$ is interpreted as the fraction of immobile protons, thus, of protons participating only to the harmonic dynamics in the material, but not to the relaxation process. This approach requires an estimation of the Debye-Waller factor, $\exp \left(-\Delta U_{\text {iso }} Q^{2}\right)$, which was carried out by following closely the averaged value of $m_{1}$ to higher $T$ as displayed in Fig. 2. Examples of such fits for the four samples are shown in the ESI. $\dagger$

In Fig. 7(c) we report the $\Gamma$ of 20In:BZO evaluated in this way. These data were parametrized with established jump diffusion models of Singwi and Sjölander (SSM), Hall and Ross (HRM), and Chudley and Elliot (CEM). ${ }^{\mathbf{2 0}, 21}$ Whereas the CEM refers to a jump process with a well defined jump length $l_{\text {CEM }}$, the SSM and HRM take a jump length uncertainty into consideration following exponential and Gaussian distributions, respectively. They are specified in the ESI. $\dagger$

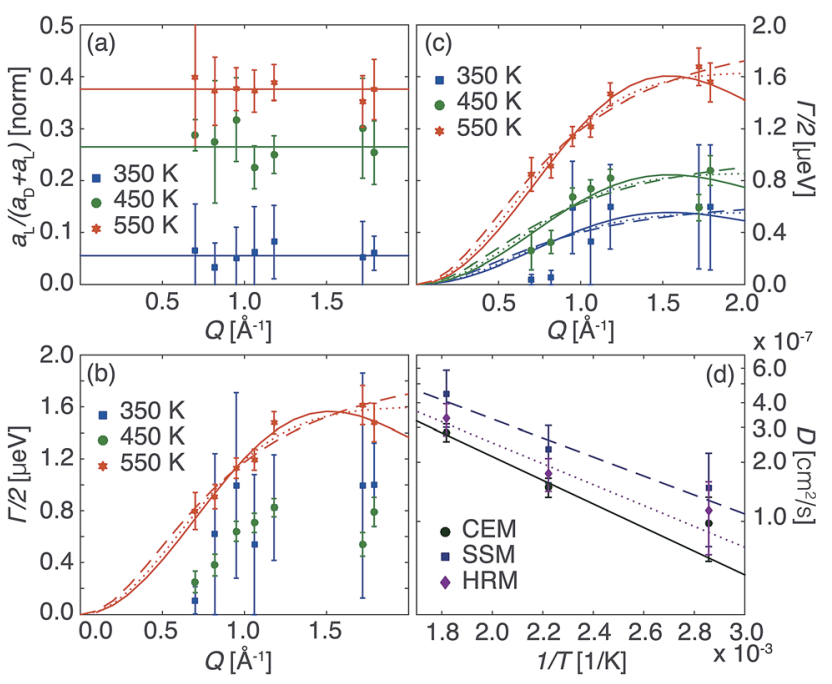

Fig. 7 Parameters derived from fitting $S(Q, \hbar \omega, T) / S^{\prime}(Q, 0,2 K)$ spectra of 20In:BZO. Intensities (a) and widths (b) of the QENS signal derived from free fits to the data with eqn (5). Solid lines in (a) indicate the average $Q$ independent values for each temperature. (c) Reports the widths of the QENS signal obtained from the fit of the spectra with $Q$-independent intensities. Lines in (b) and (c) represent the fit of the widths according to standard models of jump diffusion: CEM (solid lines), SSM (dashed lines) and HRM (dotted lines). (d) Reports the diffusion constants computed from the parameters of the jump diffusion models with the Einstein relation. Lines in (d) represent the Arrhenius fit of the diffusion coefficients. 
Table 1 Parameters from model fitting of the $S(Q, \hbar \omega)$ spectra. The jump diffusion distances $l_{C E M}, I_{S S M}$ and $l_{\text {HRM }}$ were derived as $T$-independent parameters. Activation energies $E_{\mathrm{a}}$ and characteristic trial periods $\tau_{\mathrm{o}}$ reflect the $T$ dependence of the In-doped materials

\begin{tabular}{|c|c|c|c|c|c|c|c|c|c|}
\hline & $l_{\mathrm{CEM}}[\AA]$ & $E_{\mathrm{a}}[\mathrm{meV}]$ & $\tau_{\mathrm{o}}[\mathrm{ns}]$ & $l_{\mathrm{SSM}}[\AA]$ & $E_{\mathrm{a}}[\mathrm{meV}]$ & $\tau_{\mathrm{o}}[\mathrm{ns}]$ & $l_{\mathrm{HRM}}[\AA]$ & $E_{\mathrm{a}}[\mathrm{meV}]$ & $\tau_{\mathrm{o}}[\mathrm{ns}]$ \\
\hline 10Y:BZO & $3.2(4)$ & - & - & $4(2)$ & - & - & $4(1)$ & - & - \\
\hline 10In:BZO & $3.0(4)$ & $40(9)$ & $0.22(5)$ & $2.9(4)$ & $41(11)$ & $0.13(4)$ & $2.8(6)$ & $41(10)$ & $0.17(5)$ \\
\hline 20In:BZO & $2.9(1)$ & $118(24)$ & $0.04(2)$ & $3(2)$ & $108(27)$ & $0.04(2)$ & $2.8(2)$ & $114(26)$ & $0.04(2)$ \\
\hline
\end{tabular}

Throughout all fitting approaches the CEM exhibits the most consistent results and lowest uncertainty parameters. Nonetheless, parameters such as the characteristic jump lengths and global $\Gamma$ scaling were exploited as well from the other models to characterize the diffusion process. Thereby we assumed $T$ independent jump distances for accuracy reasons of the fits. We report the derived parameters of jump distances $l_{\text {CEM }}, l_{\mathrm{SSM}}$ and $l_{\mathrm{HRM}}$, the corresponding activation energies $E_{\mathrm{a}}$ and trial periods $\tau_{\mathrm{o}}$ of the three models applied in Table 1 . The listed parameters are further exploited to compute the diffusion coefficient $D=\frac{l^{2}}{6 \tau}$ with $D$ reported in Fig. $7(\mathrm{~d})$. The full set of fit parameters is reported in the ESI. $\dagger$

The approximated jump lengths correspond approximately to the nearest oxygen-oxygen distance in BZO and its acceptordoped forms and are in agreement with results reported in the literature from various proton conducting perovskite materials. ${ }^{23-26}$ At the highest temperature the diffusion coefficients take on values very close to those reported in these studies. They approximate as well results from proton conductivity measurements. ${ }^{3,27-29}$ However, the activation energies of about 40-120 meV are smaller by a factor of 2-5 resulting in higher values towards lower $T$. The fraction of immobile protons $\varepsilon$ (not shown here) decreases systematically from 0.9 at $350 \mathrm{~K}$ for each material down to about 0.5 at $550 \mathrm{~K}$ as observed in our ToF and NSE studies. ${ }^{6,7,10}$ Both parameters, the activation energy and $\varepsilon$, are in agreement with features established through EFWS and IFWS. On one hand, the dependence of $T_{\mathrm{c}}$ upon doping level shown in Fig. 3 is reflected by the trend of the activation energy towards higher values for higher doping levels. On the other hand, the higher inelastic intensity reported in Fig. 4 is explained here by the higher number of protons $(1-\varepsilon)$ that are free to contribute to the relaxation process in the higher doped material at a given $T$.

\section{Discussion}

The different scanning modes (EFWS, IFWS, $S(Q, \hbar \omega))$ applied in this study are dedicated to shed light on the formfactor of the quasielastic signal in the different materials. They were necessary due to the distinct scattering powers of the samples and were chosen specifically for optimized signal to noise conditions and best information gain. However, the results are complementary and the same dynamic features are captured in the different response functions in an altered way.
For example, the trend of $T_{\mathrm{c}}$ computed from EFWS on the Indoped materials to increase from $200 \mathrm{~K}$ to $320 \mathrm{~K}$ with increasing $x$ is reflected in the behaviour of $E_{\text {a }}$ derived from $S(Q, \hbar \omega)$. This trend resembles that observed at a macroscopic level for activation energies derived from conductivity measurements in series of $\mathrm{Y}$ - and In-doped BZO materials. ${ }^{30,31}$ In 10Sc:BZO, 10Y:BZO and 10In:BZO the low $T_{\mathrm{c}}$ values are captured as a peak in the response of the IFWS. Along the sequence of In-doped materials this peak is shifted towards higher temperatures outside the monitored $T$ window as expected from the $T_{\mathrm{c}}$ determined from EFWS.

A distinguished $Q$ dependence of the IFWS profile in 10Sc:BZO, 10Y:BZO and 10In:BZO visualized by a shift of the peak towards lower $T$ for higher $Q$ can be explained only by a diffusion process as localized dynamics establishes a $Q$ independent peak position. The analysis of the $S(Q, \hbar \omega)$ spectra for $x=0.1$ and 0.2 In-doped BZO confirms the diffusive character of the proton dynamics and gives preference to the CEM of jump diffusion with a well defined jump length of about $3 \AA$. Thus, we conjecture that the proton dynamics responsible for the ascending intensity in the IFWS of the $x=0.2$ and 0.25 Indoped materials correspond with the dynamics of 10Sc:BZO, 10Y:BZO and 10In:BZO.

However, for 10Sc:BZO and 10Y:BZO with most distinguished peak the profile of the IFWS is not following a behaviour expected for a single jump diffusion process. Additional intensity is moved into the window at higher $T$ giving evidence of a multi-process proton relaxation. The different relaxational processes show obviously a sufficient spread in activation energies for the faster diffusion to be resolved by the here applied high-resolution technique. Having accessed comparable timescales with the NSE technique in a preceding experiment, we showed that the response of 10Sc:BZO and 10Y:BZO is rather reminiscent of a stretched exponential decay indicating the contribution of an unspecified number of relaxation processes with a certain spread of activation energies. ${ }^{10}$ The accessed $Q$ was limited to a range of 0.8-1.2 $\AA^{-1}$ in which the present IFWS data display a uniform profile for any of the samples (see Fig. 4). We conclude that the present results are in agreement with the NSE data, however, the identification of a diffusion process profits from the wider $Q$ range and higher maximum $Q$ accessed here.

We have shown as well in recent ToF studies that the short time proton relaxation in hydrated Sc-, Y-, and In-doped BZO is characterized by local proton transfers between two neighbouring oxygens and reorientations of $\mathrm{O}-\mathrm{H}$ groups. ${ }^{6,7}$ To 
substantiate this observation even higher $Q$ values of $4.5 \AA^{-1}$ had to be accessed and the energy resolution relaxed up to 0.6 meV. Thus, the time window of the present experiment is refined by more than two orders of magnitude. The fast local processes are averaged out here to a long time response reminiscent of diffusional dynamics and the formfactor of the local fast dynamics contributes to the flat background in the sampled dynamic window of $25 \pm \mu \mathrm{eV}$.

Since we deal with polycrystalline samples the monitored signal corresponds to an orientational average of the observed dynamics. Thus, it is not at hand to associate the jump lengths $\approx 3 \AA$ approximated from the different jump diffusion models with well defined translations on a crystal lattice. ${ }^{21}$ However, we may confirm that $l$ corresponds to the approximate distance between neighbouring oxygens of about $3 \AA$. If we consider that the dynamics of a proton through the BZO matrix is composed of a series of proton transfers and $\mathrm{O}-\mathrm{H}$ reorientations, as it has been elaborated by $a b$ initio computational techniques, ${ }^{32-35}$ the present experiment samples a few of those basic relaxational processes leading to an average transfer distance of an oxygenoxygen bond in BZO. Interestingly, the deviating hydration levels have only a minor influence on the characteristics of the observed proton dynamics as the features established in the different samples are in line with each other and the conclusions drawn are consistent. However, they have the disadvantageous effect of reducing the contrast of the QENS signal in respect to the elastic line. Thus, the precision of the QENS parameters derived from the analysis of $S(Q, \hbar \omega)$ is also reduced. The number of mobile protons $(1-\varepsilon)$ which shows a progressive increase upon heating is another consistent feature observed in all our QENS experiments. The important question, whether protons freed at elevated temperatures follow identical dynamics as the protons being already mobile at low $T$, remains however undisclosed from our experiments. The formfactors recorded here as well as in our prior low-resolution ToF experiments indicate that the geometry of the relaxation processes is comparable at any $T$ and for the different compounds. Thus, the differences are rather sought on the level of transition rates.

The conclusions drawn so far are in fair agreement with several QENS results discussed in the literature. However, they point as well at some differences which are worth being pointed out. The most excessive experimental attempts to characterize the microscopic proton dynamics in different doped perovskites by QENS were carried out by Hempelmann et al. ${ }^{23,24,26,36,37}$ Having examined materials such as $\mathrm{SrCe}_{0.95} \mathrm{Yb}_{0.05} \mathrm{H}_{0.02} \mathrm{O}_{2.985}$, $\mathrm{BaCa}_{1.18} \mathrm{Nb}_{1.82} \mathrm{H}_{0.2} \mathrm{O}_{8.83}$ and hydrated $\mathrm{BaZr}_{0.85} \mathrm{Me}_{0.15} \mathrm{O}_{2.925}$ with $\mathrm{Me}=\mathrm{Y}$, In and $\mathrm{Ga}$, they successfully identified a diffusive signal as well as a localized proton relaxation. The diffusive signal was approximated by a two-state model highlighting that protons move freely in dopant-free regions of the perovskite matrix with a characteristic transition rate and are trapped by the dopant cations on some longer timescale. Characteristic diffusion lengths of the two transition states approximated reasonably well the $\mathrm{O}-\mathrm{O}$ bond length and the dopant distances in the examined materials, respectively. The local relaxation process could be approximated equally well by proton transfer and reorientation of $\mathrm{O}-\mathrm{H}$ groups, respectively. By a comparable experimental protocol Pionke et al. ${ }^{38}$ reported on a local proton relaxation, and conjectured the applicability of the two-state model by qualitatively distinguishing two contributions in the QENS signal of $\mathrm{BaCa}_{1.18} \mathrm{Nb}_{1.82} \mathrm{H}_{0.2} \mathrm{O}_{8.83}$.

For a successful modelling of the QENS responses any of the above studies quantified as well an increasing number of protons participating in the relaxation upon heating. On an absolute scale the numbers correspond well to $\varepsilon$ established here and in our prior experiment. However, while the localized proton relaxation and the characterized free diffusion step found in our experimental attempts agree very well with those reports we do not identify an additional formfactor of a trapping process in our data. Braun et al. ${ }^{\mathbf{2 9 , 3 9 , 4 0}}$ made an equivalent observation of a single diffusion in ToF experiments of hydrated $\mathrm{BaCe}_{0.8} \mathrm{Y}_{0.2} \mathrm{O}_{3-x / 2}$ and $\mathrm{BaZr}_{0.9} \mathrm{Y}_{0.1} \mathrm{O}_{3-x / 2}$. Specifically, they interpreted the results in favour of a homogeneous relaxation scenario as discussed by Kreuer et al. ${ }^{3,41}$ A homogeneous relaxation should be governed by a unique activation energy rather than by two different $E_{\mathrm{a}}$ representing the free diffusion and the trapping of protons, respectively. It is indeed tempting to conjecture that our findings are as well supportive of a homogeneous relaxation rather than a two state process in particular as the activation energies associated with the QENS signal increase along the In-doping sequence $x=0.1$ to 0.2 are similar to the energies derived from the local relaxation of the differently doped BZO materials examined with ToF spectroscopy. ${ }^{5-7}$ There are, however, also differences between our data and the results reported by Braun et al. ${ }^{\mathbf{2 9 , 3 9 , 4 0}}$ The here reported formfactors feature a $Q$-independent intensity of a bulk diffusion process and favour a characteristic distance of about $3 \AA$, whereas Braun et al. ${ }^{\mathbf{2 9 , 3 9 , 4 0}}$ derived a more complex intensity profile and jump lengths of about $5 \AA$ A. The longer jump distance derived by Braun et al..$^{\mathbf{2 9 , 3 9 , 4 0}}$ is of the order of the next nearest $\mathrm{O}-\mathrm{O}$ neighbours and thus in contrast to our data as well as to results of Hempelmann et al. ${ }^{23,24,27,36,37}$ Further, the diffusion coefficients of about $D \approx 1-3 \times 10^{-7} \mathrm{~cm}^{2} \mathrm{~s}^{-1}$ (CEM) derived here are more than two orders of magnitude lower than the ones reported by Braun et $a ._{.}{ }^{\mathbf{9 , 3 9 , 4 0}}$ at ambient pressures. Interestingly, at the highest $T \approx 550 \mathrm{~K} D$ approximates very well diffusivities reported or expected for equivalent $T$ from QENS reported in ref. $24-26,36,38$ and 42 as well as from macroscopic techniques as outlined in ref. 3, 27-29 and 40. The activation energies of 40-120 meV derived from our ToF and NSE experiments are lower by a factor of 2-5 than any of the $E_{\text {a }}$ reported in the studies above. As a consequence, towards lower $T$ the diffusion coefficients derived here take on values higher than $D$ expected from these studies.

If we focus on the atomic-scale studies only, we might find physical explanations for some of the differences. In our experiments temperatures of less than $600 \mathrm{~K}$ were applied; they extend the QENS experiments discussed above towards ambient conditions. For $\mathrm{BaCe}_{0.8} \mathrm{Y}_{0.2} \mathrm{O}_{2.9}$ and $\mathrm{BaZr}_{0.9} \mathrm{Y}_{0.1} \mathrm{O}_{2.95}$, Braun et $a .^{\mathbf{2 9 , 4 0}}$ conjectured a change in proton relaxation towards dynamics governed by lower activation energies in this extended low- $T$ range. For the latter they quantified an $E_{\mathrm{a}}$ of only $40 \mathrm{meV}$, which is in good agreement with our results. 
Remarkably, it was shown as well through ab initio calculations with the doped BZO materials that a crossover from a high- $T$ to a low- $T$ regime exists. ${ }^{34,35}$ Thereby, quantum effects lower the activation energies in the low- $T$ regime to values as low as $\approx 100$ $\mathrm{meV}$ and are in very good agreement with our findings. Thus, there are arguments given in the literature indicating that the differences in $E_{\mathrm{a}}$ might have a physical significance. However, the variation in the CEM approximated formfactors of the proton diffusion reported in the different studies remain unexplained as a possible trapping effect remains elusive in our data.

The present NBS data pave a straight way to future experiments with three main goals to be targeted. Firstly, the crossover from a low- to high- $T$ behaviour of the proton dynamics requires an unequivocal determination. Higher temperatures than applied here and a wider temperature range than explored by Braun et al. ${ }^{\mathbf{2 9 , 3 9 , 4 0}}$ are required to quantify the characteristic transition temperatures in the different compounds. Secondly, the QENS formfactor awaits an accurate identification at higher $T$. On one hand, it is important to examine whether the additional relaxation process observed towards high $T$ in 10Y:BZO, 10Sc:BZO and 10In:BZO corresponds to a diffusion process, like the one identified here at lower $T$, or matches the characteristics of a localized relaxation, as observed by ToF techniques. On the other, it needs to be verified if properties are present in the highly doped compounds but are progressively shifted to higher $T$ with the doping level, as indicated by our results. Finally, a possible trapping of protons at the dopant sites as discussed by Hempelmann et al. ${ }^{23,24,26,36,37}$ requires a further elucidation. Not only higher temperatures might distinguish the trapping event from the zoo of relaxation channels characterized by NBS, NSE and ToF techniques. Experiments with dynamic windows and energy resolutions intermediate to the $1 \mu \mathrm{eV} \mathrm{NBS/NSE}$ and $200 \mu \mathrm{eV}$ ToF experiments might complement the information and offer new hints on this topic.

\section{Conclusions}

We studied the dynamics of protons in hydrated samples of the proton conducting perovskite $\mathrm{BaZr}_{1-x} \mathrm{M}_{x} \mathrm{O}$ with $\mathrm{M}=\mathrm{Sc}$ and $\mathrm{Y}$ for $x=0.1$, and $\mathrm{M}=\mathrm{In}$ for $x=0.1,0.2$ and 0.25 . By monitoring the response on an extended energy-momentum phase space with a resolution of less than $1 \mu \mathrm{eV}$ not only the onset temperatures $T_{\mathrm{c}}$ of proton relaxation were determined with high precision but also footprints of long-range proton diffusion were found. The $T_{\mathrm{c}}$ show a dependence on the type of dopant atom $\mathrm{M}$ and on the dopant level $x$, and take on values as low as about $200 \mathrm{~K}$ for the sample with $x=0.1$, increasing gradually up to above $300 \mathrm{~K}$ along the sequence of increasing $x$. As a concomitant characteristic the number of mobile protons relative to the total number of protons in the samples increases as a function of increasing temperature. The formfactor of the quasielastic signal evidencing the enhanced proton relaxation above $T_{\mathrm{c}}$ by a Lorentzian line is reminiscent of a diffusion process characterized by a $Q$-dependent width $\Gamma$ and a $Q$-independent intensity. Different standard jump diffusion models were evaluated to approximate this formfactor for the subset of $x=0.1$ and 0.2 In-doped materials. From any of the models we were able to estimate the diffusion constants $D$ and activation energies $E_{\mathrm{a}}$. In accordance with the behaviour of $T_{\mathrm{c}}, E_{\mathrm{a}}$ increases from about 40 to $120 \mathrm{meV}$ as $x$ increases from 0.1 to 0.2 . $D$ takes on values of $1-3 \times 10^{-7} \mathrm{~cm}^{2} \mathrm{~s}^{-1}$ at the highest $T$ of about $550 \mathrm{~K}$ applied. Among the different jump diffusion scenarios the model of Chudley and Elliot with a jump distance $l \approx 3 \AA$ was approximating best the recorded response.

\section{Conflicts of interest}

There are no conflicts to declare.

\section{Acknowledgements}

We are grateful for financial support from the Swedish Research Council (grant No. 2010-3519 and 2011-4887), the Swedish Foundation for Strategic Research (grant No. ICA10-0001) and the ILL (grant No. ILL1279.1). The ILL is thanked for access to neutron beam facilities (experiment 7-03-126). The group of $\mathrm{R}$. Seshadri at the UCSB and S. Eriksson at Chalmers are thanked for assistance in the preparation of the samples. R. Ammer is thanked for technical assistance at the IN16B spectrometer, and for the manufacturing of the sample cells.

\section{References}

1 J. A. Kilner and M. Burriel, Annu. Rev. Mater. Res., 2014, 44, 365.

2 Y. Yamazaki, F. Blanc, Y. Okuyama, L. Buannic, J. C. LucioVega, C. P. Grey and S. M. Haile, Nat. Mater., 2013, $12,647$.

3 K. D. Kreuer, S. Adams, W. Münch, A. Fuchs, U. Klock and J. Maier, Solid State Ionics, 2001, 145, 295.

4 M. Karlsson, Phys. Chem. Chem. Phys., 2015, 17, 26.

5 M. Karlsson, A. Matic, D. Engberg, M. E. Björketun, M. M. Koza, I. Ahmed, G. Wahnström, P. Berastegui, L. Börjesson and S. G. Eriksson, Solid State Ionics, 2009, 180, 22.

6 D. Noferini, M. M. Koza and M. Karlsson, J. Phys. Chem. C, 2017, 121, 7088.

7 D. Noferini, M. M. Koza, Z. Evenson, G. J. Nilsen, A. R. Wildes and M. Karlsson, Phys. Chem. Chem. Phys., 2018, DOI: 10.1039/C7CP07340B.

8 M. Karlsson, D. Engberg, M. E. Björketun, A. Matic, G. Wahnström, P. G. Sundell, P. Berastegui, I. Ahmed, P. Falus, B. Farago, et al., Chem. Mater., 2010, 22, 740.

9 M. Karlsson, P. Fouquet, I. Ahmed and M. Maccarini, J. Phys. Chem. C, 2010, 114, 3292.

10 D. Noferini, M. M. Koza, P. Fouquet, G. J. Nilsen, M. C. Kemei, S. M. H. Rahman, M. Maccarini, S. G. Eriksson and M. Karlsson, J. Phys. Chem. C, 2016, 120, 13963.

11 B. Frick, E. Mamontov, L. van Eijck and T. Seydel, Z. Phys. Chem., 2010, 224, 33.

12 B. Frick, J. Combet and L. van Eijck, Nucl. Instrum. Methods Phys. Res., Sect. A, 2012, 669, 7. 
13 D. Richard, M. Ferrand and J. G. Kearley, J. Neutron Res., 1996, 4, 33.

14 The MathWorks Inc., MATLAB (R2015a), The MathWorks Inc., Natick, MA, 2015.

15 W. Thomas, K. Colin, et al., GNUPLOT (5.0), 2016.

16 S. W. Lovesey, Theory of Neutron Scattering from Condensed Matter, Oxford Science Publications, Oxford, 1984.

17 G. L. Squires, Introduction to the Theory of Thermal Neutron Scattering, Dover Publications, Mineola, 1996.

18 N. W. Ashcroft and N. D. Mermin, Solid State Physics, Saunders College, Philadelphia, 1976.

19 A. Rahman, K. S. Singwi and A. Sjölander, Phys. Rev., 1962, 126, 986.

20 M. Bee, Principles and Applications in Solid State Chemistry, Biology and Materials Science, Quasielastic Neutron Scattering, Adam Hilger, Bristol and Philadelphia, 1988.

21 R. Hempelmann, Quasielastic Neutron Scattering and Solid State Diffusion, Oxford University Press, Oxford, 2000.

22 C. T. Chudley and R. J. Elliot, Proc. Phys. Soc., 1961, 77, 353. 23 Th. Matzke, U. Stimming, C. Karmonik, M. Soetratmo, R. Hempelmann and F. Güthoff, Solid State Ionics, 1996, 88, 621.

24 R. Hempelmann, Phys. B, 1996, 226, 72.

25 Ch. Karmonik, R. Hempelmann, J. Cook and F. Güthoff, Ionics, 1996, 2, 69.

26 B. Groß, C. Beck, F. Meyer, T. Krajewski, R. Hempelmann and H. Altgeld, Solid State Ionics, 2001, 145, 325.

27 K. D. Kreuer, Solid State Ionics, 1999, 125, 285.

28 K. D. Kreuer, Annu. Rev. Mater. Res., 2003, 33, 333.
29 A. Braun, S. Duval, P. Ried, J. Embs, F. Juranyi, T. Strässle, U. Stimming, R. Hempelmann, P. Holtappels and T. Graule, J. Appl. Electrochem., 2009, 39, 471.

30 D. Han, N. Hatada and T. Uda, J. Am. Ceram. Soc., 2016, 99, 3745.

31 F. Giannici, A. Longo, A. Balerna, K. D. Kreuer and A. Martorana, Chem. Mater., 2009, 21, 2641.

32 M. E. Björketun, P. Sundell, G. Wahnström and D. Engberg, Solid State Ionics, 2005, 176, 3035.

33 M. E. Björketun, P. Sundell and G. Wahnström, Phys. Rev. B: Condens. Matter Mater. Phys., 2007, 76, 054307.

34 P. Sundell, M. E. Björketun and G. Wahnström, Phys. Rev. B: Condens. Matter Mater. Phys., 2007, 76, 094301.

35 Q. Zhang, G. Wahnström, M. E. Björketun, S. Gao and E. Wang, Phys. Rev. Lett., 2008, 101, 1.

36 R. Hempelmann, C. Karmonik, T. Matzke, M. Cappadonia, U. Stimming, T. Springer and M. A. Adams, Solid State Ionics, 1995, 77, 152.

37 R. Hempelmann, M. Soetratmo, O. Hartmann and R. Wäppling, Solid State Ionics, 1998, 107, 269.

38 M. Pionke, T. Mono, W. Schweika, T. Springer and H. Schober, Solid State Ionics, 1997, 97, 497.

39 A. Braun and Q. Chen, Nat. Commun., 2016, 8, 15830.

40 Q. Chen, J. Banyte, X. Zhang, J.-P. Embs and A. Braun, Solid State Ionics, 2013, 252, 2.

41 K. D. Kreuer, Solid State Ionics, 1997, 97, 1.

42 D. Wilmer, T. Seydel and K. D. Kreuer, Solid State Ionics, 2007, 972, 15. 